\title{
Repairing the Digital Divide Can Increase the Service Divide: The Effects of Patient Portals on Kidney Allocation
}

\author{
Yeongin Kim \\ Virginia Commonwealth University \\ ykim3@vcu.edu \\ Mehmet U.S. Ayvaci \\ UT Dallas \\ mehmet.ayvaci@utdallas.edu
}

\author{
Srinivasan Raghunathan \\ UT Dallas \\ sraghu@utdallas.edu
}

\begin{abstract}
The severe shortage of organs combined with increasing demand for them characterize the outcomes for the kidney allocation process. Despite the efforts to improve the allocation of kidneys, notable inefficiencies and unequal access to available organs persist across patient populations. The goal of this study is to examine (i) whether the adoption of a patient-oriented information technology (IT), namely the patient portals, can mitigate inefficiencies in the allocation of these scarce resources (kidneys) in general; (ii) whether the adoption of patient portals magnify or alleviate the disparity issues around access to transplants. Using a rich dataset of all the kidney transplant records in the U.S. from 2011 to 2014, we show that the likelihood that the patient receives deceased donor transplant at a given point in time increases in the presence of patient portals. However, the varying impact of IT across sub-populations may indicate that the efforts to bridge the digital divide may benefit some groups of patients at the expense of other groups, leading to further disparities.
\end{abstract}

\section{Introduction}

Chronic diseases have become a significant and growing problem in the U.S. and an increasing economic burden on the U.S. healthcare system. Chronic diseases are responsible for about $70 \%$ of all deaths in the U.S, and the resulting costs exceed $86 \%$ of the total healthcare spending in the U.S [1]. Due to their increasing burden on the U.S. healthcare system, experts have examined various ways to lower costs while also improving care quality. One possible avenue for better managing chronic diseases is to increase patient involvement. When patients have the primary responsibility for identifying and conveying their goals and concerns relevant to the decision they are facing, i.e., take ownership of the medical decisions, chronic disease management can be improved [2].

Better patient engagement requires providers to share relevant information with the patients [3, 4]. With increased adoption of electronic health record (EHR) systems in clinical practices, a patient web portal connected to an EHR system is considered to be an effective technology in providing patients with access to their own health information. Patient portals provide patients with a single-point access to a variety of clinical history data such as doctor visits, lab results, chronic problems, and medication. It is argued that providing patients access to such information through a portal can lead to greater patient engagement especially in chronic diseases management because (i) chronic care requires continuous oversight of health condition, (ii) continuity of oversight and timely action heavily rely on active patient participation, and (iii) patient can make informed decisions with the help of such decision aids [3]. However, hard evidence on whether and how access to patient portals improves care outcomes is limited [5].

Prior work on the effects of patient portals on medical decisions does not focus on specific contexts that require the need for such health IT [5]. To better understand the mechanisms underlying the effects of the patient portal, it is necessary to consider conditions where the intervention (technology adoption) has a possible impact on the decision making processes. In particular, to evaluate the effectiveness of patient portals, the focus needs to be on medical decisions in which patients are more likely to be involved as in the case of chronic disease management [6]. In this study, we contribute to our understanding of the impact of patient portals on care by examining the relationship between the patient portal adoption and individual patient outcomes in a unique and relevant research context, i.e., the decisions pertaining to accepting or rejecting kidney transplantation.

Kidney transplant context is a good testbed for exploring patient portals' impact on care outcomes because of the following reasons. First, unlike many other medical decisions, kidney transplant patients often have a high degree of autonomy in choosing treatment options-continue on dialysis or get transplanted - rather than playing a subordinate role (to care providers) [7]. Therefore, patients who actively engage in care delivery can significantly change the course of treatment and the role of portals is likely to be more pronounced. Second, the decision to stay on the waiting list or to get transplanted requires patients to be aware of their health status and make complex trade-offs [7]. Specifically, the supply of kidneys is very limited while transplant success is highly uncertain and depends on patient health condition and other factors. In effect, patients need to make tough choices about whether to accept a kidney. As the patients face tough choices, a portal may become a necessity for 
them as personal health information featured in a patient portal can be useful for the patient to evaluate benefits/costs of a treatment option. Third, because patients repeatedly make these decisions until a successful transplant (about 15 times until transplant occurs), patients' understanding and involvement increase over time [8]. Given the potentials of patient portals for improving patient engagement in kidney transplant, we are interested in the question, "How does the provision of health information through patient portals affect an individual patient's transplant outcomes?"

We use patients' waiting time to receive a transplant (time to transplant) as a care outcome for our main analysis. Time to transplant is considered as an important efficiency measure in transplant service because patients' health deteriorates over time while they are waiting for a kidney transplant and a majority of patients cannot receive the treatment in time due to the limited supply of kidney donations $[8,9,10]$. Hence, time to transplant is a measure of both the quality and access performance. In addition to quantifying this outcome measure for a typical patient, we also examine disparities in the access to kidney transplant (disparities in time to transplant). Kidneys have been viewed as scarce national resources due to their highly limited supply, and providing equal access to organs to all patients is an important objective to achieve for policymakers $[9,10]$. Since there still exist notable disparities in waiting time across populations, examining unequal access to care is an important research subject for policymakers to study. Considering the uneven diffusion of IT we have observed for years [11, 12] and the possible differential impact of patient portals on different patient populations, the variation in the diffusion of patient portals may or may not impact existing disparities. Besides waiting time, we also examine other efficiency measures such as quality of kidneys received for transplant and patient/kidney survival rates after receiving a transplant as an extension of our analysis.

\section{Background}

\subsection{Patient Portal}

A patient portal is a secure website that allows patients access to health information that can be linked to EHRs maintained by care providers [6]. Through a patient portal, patients can also complete forms, schedule appointments, refill prescriptions, pay medical bills, send a message to their providers, and receive educational materials. The features of patient portals may vary, but most patient portals provide patients access to obtain their personal health information such as personal medical history and laboratory test results $[13,14]$. In this study, we focus on the main functionality of patient portals - access to health information.

The conventional view about providing information through patient portals is that patients can actively involve in care process and make more informed decisions, and in turn, the outcomes would also improve $[15,16]$. A study by [6] describes the mechanisms of patient engagement and informed decision making with access to health information through patient portals. First, such access enables patients and caregivers to engage in the utilization of the information with accurate and comprehensive insight. Second, readily accessible electronic records is a critical step toward patient empowerment since patients are unable to achieve sufficient levels of desired autonomy and self-efficacy with lack of adequate information. However, there has been insufficient evidence that supports the view that patients make more informed decisions with patient portals [5].

A potential area where patient portals can substantially improve care outcomes is chronic disease management. Chronic disease management is a patient-centered disease care which integrates screening, check-ups, monitoring and coordinating treatment, and patient education, and requires high levels of patient involvement in care processes unlike traditional paternalistic approach [17, 2]. For effective chronic disease management, engaging patients in care process by providing better information access is necessary $[17,16]$. Therefore, effective use of patient portal would lead to greater engagement of patients in chronic care process where patients can have higher levels of autonomy.

\subsection{Kidney Allocation Process}

A patient seeking deceased donor kidney first registers at a transplant center. The transplant center then conducts several tests to obtain immunological information about the patient. Once the transplant center confirms that the patient is suitable for transplant, the transplant center places the patient on a national pool of people waiting for a transplant. This list of transplant candidates (waitlist) is centrally managed by United Network of Organ Sharing (UNOS). When a kidney is donated, organ procurement organization (OPO) procures and recovers the kidney, and then finds biologically compatible patients who are registered in the waitlist. The compatible candidates are then sorted into a queue that ranks the potential candidates based on UNOS's kidney allocation policy. The ranking is given to patients mainly based on accrued waiting time, blood type compatibility, age, immunological sensitivity to the kidney, and geographical distance between the donor and the patient. The system allocates the kidney based on such ranking. When a candidate receives a kidney offer the patient should decide within a short period (typically two hours) whether to accept the offer or wait for another offer. If the patient rejects the offer, the kidney is offered to lower-ranked candidates. If no candidate accepts the offer within a few days (typically 24-36 hours), then the kidney is discarded. ${ }^{1}$

The waitlist grew substantially from around 60,000 to around 100,000 patients between 2006 and 2015 primarily due to the substantial mismatch between supply and demand for kidneys. On average, only 12,000 deceased donor transplants are performed in a year and the average waiting time is 3.6 years [18]. Despite the limited supply and the ever-increasing size of the waiting pool, a patient on average rejects 15 offers

\footnotetext{
${ }^{1}$ We refer readers to [10] for more details about the allocation process.
} 
before accepting one and getting transplanted. Typically, patients reject a kidney offer because they hope to get a better quality kidney in the future. As a result, a considerable portion of kidney donations (about 16\%) is discarded.

Organ allocation for transplant has received significant research attention in recent years. Most studies in kidney allocation focus on designing optimal policies for national kidney allocation using theoretical models [10]. Several studies examine the impact of patient decisions in the kidney allocation [19, 20, 21]. Su and Zenios explore the impact of patients' heterogeneity in preference on kidney types [19] and preference revelation on kidney types [20] in the allocation process. Ata et al. [21] consider wait-listed patients' forward-looking behavior to design optimal allocation policies. These papers take the important aspects of patients' role in designing a new allocation system. However, our paper focuses on the impact of an information aid for patients, patient portals, in outcomes resulting from the existing allocation policy.

\subsection{Expected Impacts of Patient Portals}

Decision making about accepting/ rejecting a kidney offer from a deceased donor depends on many factors that may change over time, such as a patient's health status-history, kidney quality, the composition of the patients on the waitlist $[19,8]$. It has been reported that patients often find it difficult to make an informed decision on an offer and are reluctant to consider low- or moderate-quality offers from deceased donors [22, 23, 24], which partly accounts for the long waiting time in the transplant process and kidney wastage $[8,24$, 23, 25]. Although transplant centers educate the patients about transplantation at the time of waitlisting, and they periodically (e.g., on an annual basis) interact with patients during the waiting period, the relatively long time to transplant deteriorates the effectiveness of such education efforts. In addition, a typical patients lack of health awareness remains a significant concern for an effective transplant allocation.

The adoption of health IT such as patient portals can help patients make an informed decision by providing easy access to precise and relevant health information for patients' decision making $[26,15]$. A recent survey shows that transplant patients' demand for information access is generally high and care practice in transplant shifts toward patient-centric healthcare where patients have more autonomy in decision making [7, 22]. In this aspect, information sources featured by patient portals would serve as information aids for patients and can affect their decisions to transplant. Providing better information aids, such as the information access featured in patient portals, could lead to faster utilization of donated kidneys [22, 23, 24].

Patient-oriented technology adoption can be effective in improving health outcomes, particularly among minority patients who typically have less access to technology resources. In the U.S., there exist significant disparities in care outcomes, and health literacy, defined as "the ability to obtain, process, and understand health information needed to make appropriate health decisions", is commonly associated with healthcare disparities [27]. Most reported interventions to improve care outcomes in minority groups are directed at patient education and communication [28]. A study by the Institute of Medicine reports that the use of well-designed web portals can facilitate patient communication with healthcare providers and improve patient access to healthcare services [29]. Therefore, providing patients access to comprehensive and relevant health information through a patient portal can reduce the existing disparities in care outcomes.

On the other hand, however, the adoption of a patient portal would not necessarily benefit minorities more. As reported in the digital divide literature, individuals' access to IT can be limited due to a variety of factors including race, age, gender, socio-economic status, or place of residence $[11,30]$. Even among those who already have access, there can still be a disparity in the value that IT can offer due to the inequality in individuals' ability to utilize IT [11]. This inequality leads to disparities in the outcomes from the IT investments and their use, called 'digital outcome divide' [12]. In the healthcare literature, disparities in patients' access to or use of IT has been repeatedly reported in various clinical contexts including transplant [31, 32, 33, 34, 35]. African-American patients register for the use of a web portal less frequently than other ethnic groups [33], and patients with a higher education level (college or higher) use a patient portal more frequently than those with a lower education level [35]. Health literacy has also been considered as a factor leading to the digital divide among patients [36]. In the kidney transplant context, significant disparities in the access to transplant service already exist $[9,37,38,39]$. Black, less educated patients, and low-income patients are less likely to get wait-listed and tend to have a longer waiting time to receive a transplant. Therefore, it is also possible that the digital divide that might exist in the patient population would reinforce the existing disparities in the access to the transplant service.

\section{Data and Variables for The Main Analysis}

We utilize datasets from two sources-Scientific Registry of Transplant Recipients (SRTR) Standard Analytic Files (SAF) and Healthcare Information and Management Systems Society (HIMSS) database. SRTR SAF includes all transplant records collected by Organ Procurement Transplantation Network (OPTN) on solid organ transplant candidates, donors, and recipients in U.S. since 1987 to the present. Candidate records contain waitlist status history and status justification as well as summarized biological profiles of candidates and histocompatibility results. Transplant records include transplant center profiles, recipient and donor characteristics. Pediatric candidates, candidates listed for multi-organs, candidates listed at multiple-centers are also excluded from the sample since different allocation policies apply to these groups. We restrict our observation period from 2011 to 2014 because the priority rules for kidney allocation 
changed after the implementation of Kidney Allocation System (KAS). Furthermore, SRTR started a semi-annual transplant program-specific report (PSR) on transplant activities and transplant performance of each transplant center in 2010. This report provides useful metrics for managing memberships in the transplant network and insurance membership, which might cause center-level variations related to transplant performance. We exclude transplant centers which performance was flagged as 'underperforming' by PSR from the sample during the observational period. As a result, $6.4 \%$ of observations are excluded from our sample.

Our patient portal data is derived from HIMSS database that offers a large healthcare IT adoption data including patient portal adoption at the hospital level. HIMSS database covers the majority of the U.S. hospitals with membership of the U.S. healthcare systems. HIMSS database captures variation in patient portal adoption as it provides hospitals' adoption status of patient portals as well as key functionalities featured by portals over time. The functionalities include (i) personal health records (PHR), (ii) access to medical test results (test), (iii) billing, (iv) scheduling, and (v) registration. The first two functionalities are directly related to the major features of patient portals, patient engagement and information sharing. PHR provides electronic health information to patients to help them make informed decisions, and Test is a functionality that provides patients access to their lab results [40]. The other functionalities are related to non-clinical features of patient portals as they support easy access to administrative process such as paying medical bills, scheduling an appointment, and registering to hospitals.

We merge the two datasets using CMS certification numbers and our final dataset includes a total of 42,227 transplant candidates, and 219 transplant centers. Overall,17.69\% received deceased donor transplant, $11.48 \%$ received living donor transplant, $14.38 \%$ were removed from the waiting list due to deteriorating health or death, and $53.31 \%$ of patients are censored in our dataset. The average waiting time to receive deceased donor transplant among the wait-listed patient is 4.54 years. 12.5 of the patients were able to access to a patient portal with the key functionalities (PHR and Test) during the observational period.

To study the impact of patient portals on transplant decision, we use time to deceased donor transplant as the dependent variable. Specifically, time to transplant is the time difference between the date the patient registered to the waitlist and the date that the patient was removed from the list due to transplant. If a candidate has multiple removal records on the waitlist due to temporal changes in the patient's health status (such as becoming too sick), only the last event is considered. The main independent variable is Portal-main and it is coded on a scale from 0 to 2 , which indicates no portal adoption, portal adoption with PHR, and portal adoption with both PHR and Test, respectively, in a given year. We consider PHR as the basic functionality of patient portals regarding information access while lab test results (Test) can be performed by a third-party care provider and may not exist in the transplant center's data repository.

To control for variations in patient portal features, we use a binary variable, Portal-add which takes a value of one if a transplant center adopted a patient portal with any of the non-clinical features-billing, scheduling, and registration. To account for alternative variations for the time-to-event, we use two types of control variables. We include factors related to individual patients to control for patient-level variations, following the literature on kidney transplant [41, 42]. We also include center-level controls, following reports on transplant centers' activities [43, 44]. We provide descriptive statistics in Table 1.

Table 1. Variables

and Definitions - Patient Portal on Time to Transplant

\begin{tabular}{|c|c|}
\hline Variable & Definition \\
\hline \multicolumn{2}{|c|}{ Dependent variables } \\
\hline Deceased Tx & Deceased donor kidney transplant (event of interest) \\
\hline Living $\mathrm{Tx}$ & Living donor kidney transplant \\
\hline Too Sick & Worsened health condition or death \\
\hline Other & $\begin{array}{l}\text { Other event types for removal including errors, unable to } \\
\text { contact, transplant in another country, etc }\end{array}$ \\
\hline \multicolumn{2}{|c|}{ Independent variable } \\
\hline Portal-main & $\begin{array}{l}\text { Availability of the key functionalities (PHR and lab test results) } \\
\text { to a patient through a patient portal in a given year }\end{array}$ \\
\hline \multicolumn{2}{|r|}{ 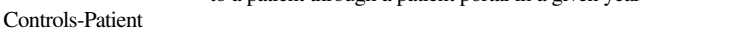 } \\
\hline Portal-add & $\begin{array}{l}\text { Availability of an additional functionality (billing, scheduling, } \\
\text { and registration) to a patient through a patient portal } \\
\text { in a given year }\end{array}$ \\
\hline Age & Age in years \\
\hline Blood Type & ABO blood type-A (reference), B, AB, O \\
\hline Gender & Biological gender of male or female (reference) \\
\hline Ethnicity & Ethnicity of White (reference), Black, Hispanic and other \\
\hline Diagnosis & $\begin{array}{l}\text { Primary cause of ESRD including hypertension (reference), } \\
\text { diabetes, polycystic kidneys, glomerulonephritis, and other }\end{array}$ \\
\hline cPRA & Calculated panel reactive antibody $(\%)$ \\
\hline Dialysis & Accumulated time spent on dialysis in years \\
\hline Pay & $\begin{array}{l}\text { Primary source for payment including public (reference) } \\
\text { and other }\end{array}$ \\
\hline Education & Higher than college degree or not (reference) \\
\hline Wait-Time & Accumulated time spent on the waitlist in years \\
\hline \multicolumn{2}{|l|}{ Controls-Center } \\
\hline Center Type & Academic or not (reference) \\
\hline NofTX20 & $\begin{array}{l}20 \text { or more transplant surgeries performed at a center } \\
\text { in a given year }\end{array}$ \\
\hline Region & OPTN 11 service regions grouped into 3 categories \\
\hline
\end{tabular}

Notes. Age, Dialysis, Wait-Time, $\mathrm{N}$ of $\mathrm{Tx}$, and Flag are time-varying variables. Region 1 includes OPTN region 6 and 8. Region 2 includes OPTN region 4,5 , and 9 . Region 0 (reference) includes the other OPTN regions.

\section{Analysis and Results}

To study the impact of patient portals on time to transplant, we use survival analysis models that analyze the time duration between an entry and exit of a subject and the effects of covariates on the time duration. Traditionally, the Kaplan-Meier model or the logit hazard model have been used for time duration analysis [45, 46, 47]. Kaplan-Meier curves indicate the proportion of patients who are still being listed on the waitlist. Logit hazard model examines incidence probability of an outcome of interest at a given time window and the hazard function to estimate covariates' impact on the incidence probability. However, these models are not appropriate since their estimates are significantly biased when observations are censored and there exist one or more events that remove a subject from the record other than the event 
of our interest, called competing events. In our analysis, patient removal from the list due to living donor transplant, bad health conditions, or some administrative errors are such competing events. To overcome this limitation, we use the cause-specific hazard model in which hazard function denotes the instantaneous rate (hazard rate) of occurrence of an event in subjects who are currently event free. The cause-specific survival model defines the hazard rate $\lambda(t, x)$ at time $t$ such that

$$
\lambda(t, x)=\lim _{d t \rightarrow 0} \frac{\operatorname{Pr}\{t \leq T<t+d t, J=j \mid T \geq t, x\}}{d t}
$$

holds, where $j \in\{1,2, \ldots, m\}$ represents one of $m$ distinct type of failure (exit of an subject), $T$ represents time duration, $x$ represents covariates.

Our regression results using the cause-specific hazard model are provided in Table 2 . We run the regression with different sets of control variables: model (1) provides univariate estimation; model (2) provides multivariate estimation without controlling hospital-level variation; model (3) provides estimation with full specification. An estimated hazard ratio (HR) in the table can be interpreted as a relative likelihood of the event of interest. For instance, if HR is greater than 1 , then it indicates a negative association between the corresponding variable and the time to transplant (i.e., a shorter time to transplant). The cause-specific hazard model requires proportional hazard rates as an assumption. We test for this assumption of each model by checking Schoenfeld residuals and verify that hazard rates do not change substantially over time (i.e., do not violate the assumption). We also check martingale residuals to confirm that the results are not driven by extreme observations.

Our models show that Portal-main is positively associated with the probability that a patient receives deceased donor transplant at a given time, regardless of model specification (models 1-3). Although there is some variation in the point estimate for Portal-main across models, we do not observe a systematic pattern to the direction of the variation, and we find the degree of the variation is small. The results imply that patients with access to personal health information through patient portals are more likely to receive a deceased donor transplant early compared to those without such access. The impact is greater when a patient portal has both PHR and Test functionalities as the coefficient of Portal-main is greatest when the value of Portal-main is 2 . This is an interesting result because the finding suggests that patients benefit more from advanced information sharing functionalities featured in a patient portal by receiving a transplant earlier. The other variable related to patient portals, Portal-add, shows clear opposite impacts on time to transplant. A possible explanation for this result is that patients with easier access to administrative services would have better access to alternative treatment options such as dialysis, and therefore the merit of choosing transplant as a treatment option could be reduced. It is also possible that patients with better access to alternatives
Table 2. Patient Portal and Time to Transplant

\begin{tabular}{|c|c|c|c|}
\hline & \multicolumn{3}{|c|}{ Hazard Ratio (Standard Error) } \\
\hline & (1) & (2) & (3) \\
\hline \multicolumn{4}{|l|}{ Portal-main } \\
\hline PHR & $1.157^{* * *}(.062)$ & $1.188 * * *(.065)$ & $1.151 * *(.065)$ \\
\hline PHR \& Test & $1.206 * * *(.045)$ & $1.200 * * *(.045)$ & $1.195 * * *(.046)$ \\
\hline Portal-add & $0.633 * * *(.017)$ & $0.690 * * *(.020)$ & $0.688 * * *(.021)$ \\
\hline Age & & $0.992 * * *(.001)$ & $0.992 * * *(.001)$ \\
\hline \multicolumn{4}{|l|}{ Blood Type } \\
\hline B & & $0.753 * * *(.032)$ & $0.765 * * *(.032)$ \\
\hline $\mathrm{O}$ & & $0.811 * * *(.025)$ & $0.819 * * *(.025)$ \\
\hline $\mathrm{AB}$ & & $0.989 \quad(.082)$ & $0.989 \quad .082$ \\
\hline Gender & & $0.934 * *(.026)$ & $0.930 * * \quad .026$ \\
\hline \multicolumn{4}{|l|}{ Ethnicity } \\
\hline Black & & $1.076^{* *} \quad(.037)$ & $1.091 * * *(.038)$ \\
\hline Hispanic & & $0.947 \quad(.038)$ & $1.089 * * *(.048)$ \\
\hline Other & & 0.997 & $1.083 * * *(.056)$ \\
\hline \multicolumn{4}{|l|}{ Diagnosis } \\
\hline Diabetes & & $0.846 * * *(.031)$ & $0.733 * * *(.030)$ \\
\hline Polycystic Kidneys & & $1.170 * * *(.071)$ & $0.733 * * *(.072)$ \\
\hline Glomer & & $1.171 * *(.073)$ & $0.733 * *(.071)$ \\
\hline Other & & $1.206 * * *(.038)$ & $1.023 \quad(.038)$ \\
\hline cPRA & & $0.515 * * *(.020)$ & $0.516 * * *(.020)$ \\
\hline Dialysis & & $1.009 * * \quad(.005)$ & $1.006 \quad(.005)$ \\
\hline Public Insurance & & $0.948 * \quad(.027)$ & $0.952 * \quad(.027)$ \\
\hline Education & & $1.054 * \quad(.029)$ & $1.059 * * \quad(.029$ \\
\hline Wait-Time & & $0.914 * * *(.007)$ & $0.931 * * *(.007)$ \\
\hline Center Type & & & $0.906 * * *(.027)$ \\
\hline NofTx20 & & & $1.271 * * *(.038)$ \\
\hline \multicolumn{4}{|l|}{ Region } \\
\hline 1 & & & $1.413 * * *(.069)$ \\
\hline 2 & & & $0.741 * * *(.025)$ \\
\hline Chi-squared & $283.38^{* * * *}$ & $1,084.820^{* * * *}$ & $1,313.35^{* * * *}$ \\
\hline Likelihood & $-58,137.288$ & $-58,024.226$ & $-58,024.226$ \\
\hline Observations & 42,227 & 42,227 & 42,227 \\
\hline
\end{tabular}

Notes. Standard errors are provided in parentheses. Robust standard errors are used. $* \mathrm{p}<0.05, * * \mathrm{p}<0.01, * * * \mathrm{p}<0.001$. Log-Pseudolikelihood is reported.

would remain healthier while waiting for transplantation (e.g., because they have better access to care through the scheduling function) and become conservative on the quality of kidney being offered, which could prolong the waiting time.

Regarding control variables, we observe consistent results from the findings in the transplant literature. Patient biological characteristics and center types are the major factors that predict time to transplant. The cPRA score has the most significant and greatest impact on the time to transplant. A $1 \%$ increase in cPRA score reduces the hazard rate by 48.5\%. This finding is expected since high cPRA implies a less likelihood of finding a matching kidney from the national pool. Interestingly, education and public insurance are also significant predictors. The hazard rate for patients with a bachelor degree or higher is $5.9 \%$ higher than the hazard rate for patients with a high school degree or lower. This socioeconomic disparity can possibly be due to the relationship between patients and hospitals. There might exist communication barriers between hospitals and patients, and it would be easier to address the barriers in higher education groups. Inefficient communication is a strong predictor of medical non-adherence, and medical non-adherence of a patient can lead to a period of probation and a temporary removal of the patient from the waiting list [48]. The hazard rate for patients with a public insurance as the primary source 
of payment is $6.9 \%$ lower than the hazard rate for those with other payment types. This finding can be explained by the current Medicare policies for transplant which require a high copayment rate (about 20\%) for immunosuppression and covers only 36 months after transplantation. Therefore, the patients would expect a financial burden if they consider transplant and postpone their decision on transplant [41].

As a robustness check, we consider alternative model specifications with the following econometric concerns. First, our current model does not account for time trend in overall waiting time. The number of wait-listed patients has steadily increased while the kidney donation has remained consistent during the observational period [49]. This trend might impact patients' decision on a kidney offer because the composition of the patients on the waitlist also affect a patient's decision on a kidney offer. Therefore, it might be reasonable to include an additional control variable that can account for the time-related variation. We include a linear time trend as the control in the cause-specific hazard model and provide the results. Second, there might exist unobserved heterogeneity that could create a bias in the results. The unobserved heterogeneity can be addressed by the inclusion of fixed or random effects in the models. However, the cause-specific hazard model does not allow for using fixed effects and allow only for random effects model called frailty model. We re-estimate the impact of patient portals by using the frailty model. Third, subdistribution hazard model introduced by Fine and Gray $(\mathrm{F} / \mathrm{G})$ is another feasible model for survival analysis with competing events [50]. Although we use the cause-specific model due to its ease of use and simple implementation, subdistribution hazard model would be more useful if our interest is to predict the probability of an event (transplant) within a period [51]. We run the subdistribution hazard model with the same set of variables. Forth, we use Meaningful Use criteria as an alternative measure for the key functionalities of patient portals. Meaningful Use is an incentive program governed by CMS and participating providers (hospitals or physicians). In this program, providers can receive rewards if they use a CMS-certified health IT and met IT usage criteria specified in the program. Meaningful Use criteria require care providers to electronically share personal health information with their patients upon request, and we expect Meaningful Use criteria would have a similar impact as the key functionalities of patient portals. We replace Portal-main and Portal-add with a new variable, $M U$, which is coded as 1 if the hospital met MU criteria and 0 otherwise. We report the regression results with different specifications in Table 3. We find that our findings remain consistent, qualitatively.

Note that our main independent variable measures adoption of patient portals rather than actual usage of it and we implicitly assume that patient portals are useful in evaluating 'complex trade-offs' required to make the decision on transplant. We use two approaches to check the impact of this assumption on our analysis. First, we estimate the impact of patient portals only on two subsamples-patients whose cPRA score is greater than $80 \%$ and patients whose Estimated
Table 3. Analysis Using Alternative Models

\begin{tabular}{lcccc}
\hline & \multicolumn{4}{c}{ Hazard Ratio (Standard Error) } \\
\cline { 2 - 5 } & (1) Time Trend & (2) Frailty & (3) F/G & $(4) \mathrm{MU}$ \\
\hline Portal-main & & & & \\
$\quad$ PHR & $1.215^{* * *}(.069)$ & $1.172^{* * *}(.067)$ & $1.149^{* * *}(.065)$ & \\
PHR \& Test & $1.281^{* * *}(.050)$ & $1.176^{* * *}(.046)$ & $1.200^{* * * *}(.047)$ & \\
Portal-add & $0.903^{* *}(.031)$ & $0.992^{* * *}(.001)$ & $0.700^{* * * *}(.021)$ & \\
MU & & & & $1.751^{* * * *}(.049)$ \\
Patient Controls & Yes & Yes & Yes & Yes \\
Center Controls & Yes & Yes & Yes & Yes \\
\hline \hline Chi-squared & $2,160.07 * * *$ & $1,118.19^{* * * *}$ & $1,338.91^{* * *}$ & $1,518.24 * * *$ \\
Likelihood & $-57,785.579$ & $-57,996.357$ & $-58,586.012$ & $-57,894.88$ \\
Observations & 42,227 & 42,227 & 42,227 & 42,227 \\
\hline
\end{tabular}

Notes. Standard errors are provided in parentheses. Robust standard errors are used. $* \mathrm{p}<0.05,{ }^{*} * \mathrm{p}<0.01, * * * \mathrm{p}<0.001$. Log-Pseudolikelihood is reported.

Post Transplant Survival (EPTS) score is greater than $80 \%$. The EPTS score measures estimated years of kidney function after transplant and a high EPTS score implies shorter years of kidney function after transplant. Patients with a high cPRA score have a significantly lower chance of getting a kidney offer by the allocation system and have a longer waiting time in general. Therefore, patients with a high cPRA score would more likely to accept a kidney offer immediately and less likely to consider staying on the waiting list. On the other hand, however, patients with a high EPTS score would expect less benefits from receiving transplant unless a high quality kidney becomes available to them, which may prolong the waiting time. Second, we replace our dependent variable, time to deceased donor transplant, with time to living donor transplant. Living donor transplant yields much better post-transplant outcomes than deceased donor transplant and most patients strongly prefer kidney offers from living donors over kidney offers from deceased donors. Therefore, since a decision on a living kidney offer does not require evaluation of the complex 'trade-offs', we should not observe a significant impact of information access features of patient portals (PHR and Test) in time to living donor transplant. Our expectations are verified in Table 4 . We cannot find any evidence that contradicts our assumption.

Table 4. Verification of Underlying Assumptions

\begin{tabular}{lccc}
\hline & \multicolumn{3}{c}{ Hazard Ratio (Standard Error) } \\
\cline { 2 - 4 } & (1) cPRA $>80 \%$ & (2) EPTS $>80 \%$ & (3) Living Tx \\
\hline Portal-main & $1.214(.178)$ & $1.137(.135)$ & $0.783(.188)$ \\
PHR & $1.166(.126)$ & $1.073(.094)$ & $0.918(.127)$ \\
PHR \& Test & $0.704^{* * *}(0.056)$ & $0.692^{* * *}(.024)$ & $0.824^{\Delta}(.021)$ \\
Portal-add & Yes & Yes & Yes \\
Patient Controls & Yes & Yes & Yes \\
Center Controls & $703.56^{* * *}$ & $305.56^{* * *}$ & $590.21^{* * *}$ \\
\hline \hline Chi-squared & $-6,337.370$ & $-11,598.49$ & $-4,613.412$ \\
Likelihood & 9,707 & 12,446 & 42,227 \\
Observations &
\end{tabular}

Notes. Standard errors are provided in parentheses. Robust standard errors are used. $\Delta \mathrm{p}<0.1, * \mathrm{p}<0.05, * * \mathrm{p}<0.01, * * * \mathrm{p}<0.001$.

Log-Pseudolikelihood is reported. 


\subsection{Stratified Analysis by Ethnicity and Education}

In general, the distribution of IT accessibility and usability are known to be significantly different for different ethnicity and socioeconomic groups [52, 12, 30]. This phenomenon called the 'Digital Divide,' has been repeatedly reported by research studying health IT. The difference in the distribution of patients' Internet use and access is attributed to ethnicity and level of education [32, 31]. Despite the high Internet accessibility, African-American patients enroll in a patient portal less frequently than white patients $[33,34]$, and patients with a college degree or higher use a patient portal more frequently than those with a high school degree or lower [35]. In that sense, the impact of patient portals on transplant outcomes can vary depending on a patient's ethnicity and education status. We test this expectation by performing stratified analysis with the cause-specific hazard model. We first analyze by two ethnicity groups, African-American and other. Then, we analyze by education level, a patient having a college degree or higher and other.

We report the results from the stratified analysis in Table 5. Column (1a) and (1b) provide the estimation results for African-American patients and others, respectively. The impact of patient portals is not significant for patients with African-American ethnicity while it is significant for those with other ethnicities. The model stratification test confirms the validity of this stratified model ( $\left.\chi^{2}=9.64, d f=1, p<0.01\right)$. Column ( $\left.2 \mathrm{a}\right)$ and ( $\left.2 \mathrm{~b}\right)$ provide the estimation results for patients with a higher education level (patients with a college degree or higher) and patients with a lower education level (patients with a high school degree or lower), respectively. The impact of patient portals is not significant for patients with African-American ethnicity when patient portals are equipped only with the basic key feature, $P H R$. In contrast, the impact is significant for higher education group. The model stratification test also confirms the validity of this stratified model $\left(\chi^{2}=5.06, d f=1, p<0.1\right)$. To summarize, the results show that the impact of patient portals on time-to-transplant varies depending on a patient's ethnicity and educational status.

Table 5. Stratified Analysis by Ethnicity and Education

\begin{tabular}{|c|c|c|c|c|}
\hline & \multicolumn{4}{|c|}{ Hazard Ratio (Standard Error) } \\
\hline & $\begin{array}{l}\text { (1a) African } \\
\text {-American }\end{array}$ & (1b) Others & $\begin{array}{l}\text { (2a) Education } \\
\text { Low }\end{array}$ & $\begin{array}{l}\text { (2b) Education } \\
\text { High }\end{array}$ \\
\hline \multicolumn{5}{|l|}{ Portal-main } \\
\hline PHR & $0.969(.077)$ & $1.343 * * *(.107)$ & $1.078 \quad(.069)$ & $1.459 * *(.171)$ \\
\hline PHR \& Test & $0.998(.064)$ & $1.305^{* * *}(.064)$ & $1.177 * * *(.051)$ & $1.249 * * \quad(.111)$ \\
\hline Portal-add & $0.723 * * *(.034)$ & $0.678 * * *(.026)$ & $0.694 * * *(.023)$ & $0.672 * * *(.050)$ \\
\hline Patient Controls & Yes & Yes & Yes & Yes \\
\hline Center Controls & Yes & Yes & Yes & Yes \\
\hline Chi-squared & $737.85^{* * * *}$ & $747.85^{* * * *}$ & $926.97 * * *$ & $406.93 * * *$ \\
\hline Likelihood & $-21,095.941$ & -32.995 .331 & $-58,586.012$ & -10.232 .53 \\
\hline Observations & 16,831 & 25,446 & 31,851 & 10,426 \\
\hline
\end{tabular}

Notes. Standard errors are provided in parentheses. Robust standard errors are used. $* \mathrm{p}<0.05, * * \mathrm{p}<0.01, * * * \mathrm{p}<0.001$. Log-Pseudolikelihood is reported.

\subsection{Analysis Using Alternative Care Outcomes}

In this section, we examine the impact of patient portals on alternative care outcomes-quality of the kidney accepted for transplant and the survival rate of a patient after transplant. Time to transplant is an efficiency measure for transplant services. The supplemental analysis is worth conducting because if the reduction in time to transplant is achieved at the expense of sacrificing the quality of kidney, it indicates a trade-off patients are facing from the overall process perspective. Regarding the kidney quality, we use 'expanded criteria donor (ECD)' and 'kidney donor profile index (KDPI).' ECD is a measure for overall quality of a donated kidney, and a donated kidney is marked as ECD if the donor is old and/or has more than two risks factors that negatively affect the recipient's survival after transplant. KDPI is a newly introduced measure in 2014 with the change in the allocation system. KDPI combines a variety of donor factors into a single number (ranges from $0 \%$ to $100 \%$ ) that summarizes the likelihood of kidney failure after transplant. We coded KDPI as 1 if the KDPI score of a kidney is less than $20 \%$ (good quality kidney) and 0 otherwise. We use a $20 \%$ threshold since it is used for the current allocation policies to identify good quality kidneys. ${ }^{2}$ For patient/kidney survival rates, we use 1-year patient mortality rate (PMR) and 1-year kidney failure rate (KFR) as they are widely used measures for policy oversight for kidney transplant programs (OPTN, SRTR).

We first run logit regressions to estimate the impact of patient portals on ECD and KDPI with the same set of control variables. We then run another set of logit regressions to estimate the impact of patient portals on PMR and KFR. The data we use for these regressions is a cross-sectional data that snapshots all the variables at the time patients exit from the observation due to deceased-donor transplant. We use a different set of control variables as some operational factors are known to affect the survival rates and some of the variables we used in the previous analysis are known not affect the survival rates (descriptions not reported due to space restrictions). We report the regression results in Table 6. Column (1a), (1b), (2a), and (2b) provide the coefficient of Portal-main on ECD, KDPI, PMR, and KFR, respectively. We cannot find evidence that patient portal adoption with the key functionalities has a negative impact on those alternative care outcomes. We also conducted a stratified analysis to examine the varying impact of patient portals by ethnicity and education using the alternative outcomes. The results of the analysis provide no significant evidence.

\section{Discussion and Conclusion}

In this paper, we study the role of a patient's access to health information featured in a patient portal in the national kidney allocation process. Our study shows that a patient's access to health information featured in a patient portal can

\footnotetext{
${ }^{2}$ For further information, we refer readers to
ws://optn.transplant.hrsa.gov.
} 
Table 6. Analysis Using Alternative Care Outcomes

\begin{tabular}{|c|c|c|c|c|}
\hline & \multicolumn{4}{|c|}{ Odd Ratio (Standard Error) } \\
\hline & (1a) ECD & (2b) $\mathrm{KDPI} \leq 20$ & (2a) PMR & (2b) KFR \\
\hline \multicolumn{5}{|l|}{ Portal-main } \\
\hline PHR & $1.109(.148)$ & $0.994 \quad(.108)$ & $0.612(.194)$ & $1.212(.315)$ \\
\hline PHR \& Test & $0.927(.095)$ & $1.271 * *(.097)$ & $1.334(.238)$ & $0.983(.215)$ \\
\hline Portal-add & $-0.036(.076)$ & $-0.104^{\Delta} \quad(.058)$ & $0.076(.164)$ & $0.045(.160)$ \\
\hline Patient Controls & Yes & Yes & Yes & Yes \\
\hline Center Controls & Yes & Yes & Yes & Yes \\
\hline Chi-squared & 729.77**** & $257.54 * * *$ & $128.68 * * *$ & $136.26 * * *$ \\
\hline Likelihood & $-2,782.927$ & -4.153 .325 & -914.358 & -842.121 \\
\hline Observations & 7,744 & 7,744 & 7,744 & 7,744 \\
\hline
\end{tabular}

Notes. Standard errors are provided in parentheses. Robust standard errors are used. $\Delta \mathrm{p}<0.1, * \mathrm{p}<0.05, * * \mathrm{p}<0.01, * * * \mathrm{p}<0.001$

Log-Pseudolikelihood is reported.

reduce the time to receiving a kidney transplant. This result can be explained by the impact of information availability in patients' likelihood of accepting kidney offers. Our study also shows that the impact of the information access is limited or insignificant in some subpopulations where patients tend to have a longer waiting time, which may imply further disparities in the transplant service. These findings have important practical implications at multiple levels: (i) at the patient level from a chronic disease management perspective, (ii) at the organizational level from a business efficiency perspective for a transplant center, and (iii) at the societal level from a resource allocation perspective. We describe each perspective next.

First of all, although patient portals tethered to a hospital's IT system have been touted for better management of chronic diseases, there has been little conclusive empirical research to support the view that patients make more informed decisions with patient portals and the outcomes would also improve [5]. We believe our research can help in directing future efforts to improve patient management for chronic diseases by harnessing the power of patient-oriented health IT. Relative to other settings used in the existing literature, our research setting is uniquely positioned to examine the impact of health IT in chronic disease management because (i) patients can have high degree of autonomy in making health decisions related to kidney transplant and (ii) a rich dataset that includes activities of all transplant patients in the U.S. exists.

Second, management of transplant candidates (or wait-listed patients) requires substantial effort because transplant centers need to frequently communicate with patients, conduct routine medical tests, and educate patients. With the increasing number of candidates for deceased-donor kidney transplant, waitlist management becomes a significant challenge for transplant centers. Failing to manage waitlist properly can decrease a patient' chance of receiving a transplant and increases a patient' mortality rate while on the waitlist. A reduced transplant rate and increased waitlist mortality can significantly deteriorate the financial soundness of a transplant program. Our findings imply that patient portal implementation can facilitate the rapid placement of donated kidneys, improve post-transplant outcomes, reduce the workload for waitlist management for a deceased-donor kidney transplant. However, the impacts may be limited to specific patient groups such as highly educated or non-African-American patients, which requires transplant hospitals to customize their strategies when implementing patient portals as an intervention for improving the patient management.

Third, since the passage of the National Organ Transplant Act (NOTA) in 1984, donated kidneys have been viewed as national resources because of the limited supply of donated kidneys and the ever-increasing demand for transplants. Any improvement in the efficiency of allocation would have a significant impact on social welfare. Our study demonstrates that patient portals have positive impacts in reducing time to transplant for deceased-donor transplants while not hurting other efficiency outcomes. However, since the impact could vary on subpopulations, the efforts to bridge the digital divide may benefit some patient groups at the expense of other groups, leading to further disparities in the care service. Our analysis of multiple factors provides a more clear picture for a better policy-making to reduce the existing disparities in the kidney transplant context. Specifically, policymakers can utilize our findings on specific functionalities in the patient portal, patient factors (e.g., education), and post-transplant outcomes (e.g., graft failure rate) when making adjustments to policy on kidney allocation system and the federal health IT policies.

The growing trend of patient portal adoption for chronic disease management has prompted significant academic research on the effectiveness of patient portals [5]. However, few studies have investigated whether patient portals affect care decisions and outcomes. The conventional view is that when patients make more informed decisions with the help of such decision aids, the outcomes would also improve $[15,16]$. However, there has been little conclusive empirical research to support the claim [5]. The implications of the studies on patient portals typically are limited due to a small sample size or relatively short observation periods. Therefore, the evidence about effect of patient portals on patients' decisions is insufficient to draw a conclusion as previous studies do not focus on specific contexts that require the need for such health IT $[5,6]$. We extend the research on patient portals by examining the relation between the portal intervention and care outcomes by utilizing a rich panel dataset in a unique and relevant research context. Also, most of these studies on patient portals use a binary variable to capture the portal intervention rather than using a continuous variable (i.e. different adoption levels), which may underestimate the value of patient portals. In contrast, our study uses a more granular measure of adoption by focusing on the key functionalities of patient portals in evaluating the impact of information access on care outcomes.

Our study also contributes to the discussion on digital outcome divide. Digital outcome divide is a goods-centric view of digital divide and it refers to a disparity in the value that IT offers due to an uneven diffusion of IT across individuals at different socioeconomic levels or demographic 
categories [12]. This outcome-dominant perspective is appropriate when the supply of final goods or services is highly limited and the even distribution of final goods or services is required by the society [53]. Because of scarcity of kidney donation and the ever-increasing size of the demand pool, donated kidneys haven viewed as national resources. Therefore, given the different distribution of the value IT that has been reported in various areas, it is a valid and meaningful to ask whether bridging the digital divide (portal adoption) leads to service divide in kidney transplant context. Our findings on varying impact of patient portals across populations can extend the digital divide literature to the societal improvements on service innovation [54].

Our study has a number of limitations and can be extended in several directions. First of all, our dataset has two limitations. We cannot observe patients' actual usage of a patient portal from our dataset. Also, we cannot observe individual patients' history of kidney offers. The use of a dataset that contains portal usage and offer history would provide better contextual insights of the value of patient portals and the mechanisms underlying the effects of patient portals. Moreover, decisions on portal adoption could be endogenous, which may overestimate the benefits from portal adoption by weakening our casual inference. For linear models, the use of instrumental variables (IVs) or matching can partially address this identification challenge. However, IVs and matching approaches are not feasible for most of traditional survival models for competing events including the models used in this study. A few recent studies discuss about the application of the two approaches [55, 56], and we may address the econometric concern following their suggestions.

\section{References}

[1] CDC, "CDC's chronic disease prevention system." Retrieved April 17, 2017, http://www.cdc.gov/ chronicdisease/about/prevention.htm, 2014.

[2] T. Bodenheimer, K. Lorig, H. Holman, and K. Grumbach, "Patient self-management of chronic disease in primary care," The Journal of the American Medical Association, vol. 288, no. 19, pp. 2469-2475, 2002.

[3] S. Emont, "Measuring the impact of patient portals: what the literature tells us." Retrieved April 17, 2017, http://www.chcf.org/ /media/MEDIA\% 20LIBRARY\%20Files/PDF/PDF\%20M/PDF\% 20MeasuringImpactPatientPortals.pdf, 2011.

[4] T. Irizarry, A. D. Dabbs, and C. R. Curran, "Patient portals and patient engagement: a state of the science review," Journal of Medical Internet Research, vol. 17, no. 6, 2015.

[5] C. L. Goldzweig, G. Orshansky, N. M. Paige, A. A. Towfigh, D. A. Haggstrom, I. Miake-Lye, J. M. Beroes, and P. G. Shekelle, "Electronic patient portals: evidence on health outcomes, satisfaction, efficiency, and attitudes: a systematic review," Annals of Internal Medicine, vol. 159, no. 10, pp. 677-687, 2013

[6] T. Otte-Trojel, A. de Bont, T. G. Rundall, and J. van de Klundert, "How outcomes are achieved through patient portals: a realist review," Journal of The American Medical Informatics Association, vol. 21, no. 4, pp. 751-757, 2014.
[7] D. A. Solomon, "Decision making by patients awaiting kidney transplant (Doctoral dissertation)." Retrieved April 17, 2017, https://elischolar.library.yale. edu/cgi/viewcontent.cgi?referer=https: //scholar.google.com/\&httpsredir=1\& article=1094\&context=ymtdl, 2010.

[8] J. Zhang, "The sound of silence: Observational learning in the us kidney market," Marketing Science, vol. 29, no. 2, pp. 315-335, 2010.

[9] B. Ata, A. Skaro, and S. Tayur, "Organjet: Overcoming geographical disparities in access to deceased donor kidneys in the united states," Management Science, vol. 63, no. 9, pp. 2776-2794, 2016.

[10] D. Bertsimas, V. F. Farias, and N. Trichakis, "Fairness, efficiency, and flexibility in organ allocation for kidney transplantation," Operations Research, vol. 61, no. 1, pp. 73-87, 2013.

[11] F. J. Riggins and S. Dewan, "The digital divide: Current and future research directions," Journal of the Association for information systems, vol. 6, no. 12, p. 13, 2005.

[12] K.-K. Wei, H.-H. Teo, H. C. Chan, and B. C. Tan, "Conceptualizing and testing a social cognitive model of the digital divide," Information Systems Research, vol. 22, no. 1, pp. 170-187, 2011.

[13] S. N. Weingart, D. Rind, Z. Tofias, and D. Z. Sands, "Who uses the patient internet portal? the patientsite experience," Journal of the American Medical Informatics Association, vol. 13, no. 1, pp. 91-95, 2006.

[14] A. Baird, M. F. Furukawa, and T. Raghu, "Understanding contingencies associated with the early adoption of customer-facing web portals," Journal of Management Information Systems, vol. 29, no. 2, pp. 293-324, 2012.

[15] A. Al-Busaidi, A. Gray, and N. Fiddian, "Personalizing web information for patients: linking patient medical data with the web via a patient personal knowledge base," Health Informatics Journal, vol. 12, no. 1, pp. 27-39, 2006.

[16] F. J. Fowler, C. A. Levin, and K. R. Sepucha, "Informing and involving patients to improve the quality of medical decisions," Health Affairs, vol. 30, no. 4, pp. 699-706, 2011.

[17] A. S. Young, E. Chaney, R. Shoai, L. Bonner, A. N. Cohen, B. Doebbeling, D. Dorr, M. K. Goldstein, E. Kerr, P. Nichol, et al., "Information technology to support improved care for chronic illness," Journal of General Internal Medicine, vol. 22, no. 3, pp. 425-430, 2007.

[18] United States Renal Data System (USRDS), "2016 USRDS annual data report: Epidemiology of kidney disease in the United States," 2016.

[19] X. Su and S. Zenios, "Patient choice in kidney allocation: The role of the queueing discipline," Manufacturing \& Service Operations Management, vol. 6, no. 4, pp. 280-301, 2004.

[20] X. Su and S. A. Zenios, "Recipient choice can address the efficiency-equity trade-off in kidney transplantation: A mechanism design model," Management Science, vol. 52, no. 11, pp. 1647-1660, 2006.

[21] B. Ata, Y. Ding, and S. A. Zenios, "Kdpi-dependent ranking policies: Shaping the allocation of deceased-donor kidneys in the new era," Working Paper, 2017.

[22] M. L. Volk, R. S. Tocco, S. J. Pelletier, B. J. Zikmund-Fisher, and A. S. Lok, "Patient decision making about organ quality in liver transplantation," Liver Transplantation, vol. 17, no. 12, pp. 1387-1393, 2011.

[23] E. J. Gordon, E. Reddy, D. P. Ladner, J. Friedewald, M. M. Abecassis, and M. G. Ison, "Kidney transplant candidates understanding of increased risk donor kidneys: A qualitative study," Clinical Transplantation, vol. 26, no. 2, pp. 359-368, 2012 . 
[24] R. L. Ros, L. M. Kucirka, P. Govindan, H. Sarathy, R. A. Montgomery, and D. L. Segev, "Patient attitudes toward cdc high infectious risk donor kidney transplantation: inferences from focus groups," Clinical Transplantation, vol. 26, no. 2 , pp. 247-253, 2012

[25] A. D. Waterman, A. C. Barrett, and S. L. Stanley, "Optimal transplant education for recipients to increase pursuit of living donation," Progress in Transplantation, vol. 18, no. 1 pp. 55-62, 2008.

[26] A. Kumar and R. Telang, "Does the web reduce customer service cost? empirical evidence from a call center," Information Systems Research, vol. 23, no. 3-part-1, pp. 721-737, 2012.

[27] D. A. Kindig, A. M. Panzer, L. Nielsen-Bohlman, et al., Health literacy: a prescription to end confusion. National Academies Press, 2004.

[28] H. People, "Health communication and health information technology." Retrieved September 2, 2018, http://www.healthypeople.gov/2020/ topicsobjectives2020/overview. aspx, 2010.

[29] A. Nelson, "Unequal treatment: confronting racial and ethnic disparities in health care.," Journal of the National Medical Association, vol. 94, no. 8, p. 666, 2002.

[30] R. Agarwal, A. Animesh, and K. Prasad, "Research notesocial interactions and the digital divide: Explaining variations in internet use," Information Systems Research, vol. 20, no. 2, pp. 277-294, 2009.

[31] M. Lockwood, M. Saunders, M. A. Josephson, Y. T. Becker, and C. Lee, "Determinants of frequent internet use in an urban kidney transplant population in the united states: characterizing the digital divide," Progress in Transplantation, vol. 25, no. 1 , pp. 9-17, 2015.

[32] M. Brodie, R. E. Flournoy, D. E. Altman, R. J. Blendon, J. M. Benson, and M. D. Rosenbaum, "Health information, the internet, and the digital divide," Health Affairs, vol. 19, no. 6, pp. 255-265, 2000.

[33] C. K. Yamin, S. Emani, D. H. Williams, S. R. Lipsitz, A. S Karson, J. S. Wald, and D. W. Bates, "The digital divide in adoption and use of a personal health record," Archives of Internal Medicine, vol. 171, no. 6, pp. 568-574, 2011.

[34] M. S. Goel, T. L. Brown, A. Williams, A. J. Cooper, R. Hasnain-Wynia, and D. W. Baker, "Patient reported barriers to enrolling in a patient portal," Journal of the American Medical Informatics Association, vol. 18, no. Supplement_1, pp. i8-i12, 2011.

[35] U. Sarkar, A. J. Karter, J. Y. Liu, N. E. Adler, R. Nguyen, A. López, and D. Schillinger, "Social disparities in internet patient portal use in diabetes: evidence that the digital divide extends beyond access," Journal of the American Medical Informatics Association, vol. 18, no. 3, pp. 318-321, 2011.

[36] E. Neter and E. Brainin, "eHealth literacy: Extending the digital divide to the realm of health information," Journal of Medical Internet Research, vol. 14, no. 1, 2012.

[37] E. J. Gordon, D. P. Ladner, J. C. Caicedo, and J. Franklin, "Disparities in kidney transplant outcomes: a review," in Seminars in Nephrology, vol. 30, pp. 81-89, Elsevier, 2010.

[38] R. E. Patzer, S. Amaral, H. Wasse, N. Volkova, D. Kleinbaum, and W. M. McClellan, "Neighborhood poverty and racial disparities in kidney transplant waitlisting," Journal of the American Society of Nephrology, vol. 20, no. 6, pp. 1333-1340, 2009.

[39] L. M. Kucirka, M. Grams, K. S. Balhara, B. G. Jaar, and D. L. Segev, "Disparities in provision of transplant information affect access to kidney transplantation," American Journal of Transplantation, vol. 12, no. 2, pp. 351-357, 2012.
[40] Healthcare Information and Management Systems Society, HIMSS dictionary of health information technology terms, acronyms, and organizations. CRC Press, 2017.

[41] D. A. Axelrod, N. Dzebisashvili, M. A. Schnitzler, P. R. Salvalaggio, D. L. Segev, S. E. Gentry, J. Tuttle-Newhall, and K. L. Lentine, "The interplay of socioeconomic status, distance to center, and interdonor service area travel on kidney transplant access and outcomes," Clinical Journal of The American Society of Nephrology, pp. CJN-04940610, 2010.

[42] F. Torlak, M. Ayvaci, M. Ahsen, C. Arce, M. Vazquez, and B. Tanriover, "Estimating waiting time for deceased donor renal transplantion in the era of new kidney allocation system," in Transplantation Proceedings, vol. 48, pp. 1916-1919, Elsevier, 2016.

[43] B. Kasiske, N. Salkowski, A. Wey, A. Israni, and J. Snyder, "Potential implications of recent and proposed changes in the regulatory oversight of solid organ transplantation in the united states," American Journal of Transplantation, vol. 16, no. 12, pp. 3371-3377, 2016.

[44] R. Patzer, L. Plantinga, J. Krisher, and S. Pastan, "Dialysis facility and network factors associated with low kidney, transplantation rates among united states dialysis facilities," American Journal of Transplantation, vol. 14, no. 7, pp. 1562-1572, 2014.

[45] S. Li, J. Shang, and S. A. Slaughter, "Why do software firms fail? capabilities, competitive actions, and firm survival in the software industry from 1995 to 2007,' Information Systems Research, vol. 21, no. 3, pp. 631-654, 2010.

[46] O. Temizkan, R. L. Kumar, S. Park, and C. Subramaniam, "Patch release behaviors of software vendors in response to vulnerabilities: an empirical analysis," Journal of management Information Systems, vol. 28, no. 4, pp. 305-338, 2012.

[47] G. Hoetker, "The use of logit and probit models in strategic management research: Critical issues," Strategic Management Journal, vol. 28, no. 4, pp. 331-343, 2007.

[48] E. S. Schaeffner, J. Mehta, and W. C. Winkelmayer, "Educational level as a determinant of access to and outcomes after kidney transplantation in the united states," American Journal of Kidney Diseases, vol. 51, no. 5, pp. 811-818, 2008.

[49] A. Hart, J. Smith, M. Skeans, S. Gustafson, D. Stewart, W. Cherikh, J. Wainright, A. Kucheryavaya, M. Woodbury, J. Snyder, et al., "OPTN/SRTR 2015 annual data report: kidney," American Journal of Transplantation, vol. 17, no. S1, pp. 21-116, 2017.

[50] J. P. Fine and R. J. Gray, "A proportional hazards model for the subdistribution of a competing risk," Journal of the American Statistical Association, vol. 94, no. 446, pp. 496-509, 1999.

[51] D. G. Kleinbaum, "Survival analysis, a self-learning text," Biometrical Journal, vol. 40, no. 1, pp. 107-108, 1998.

[52] J. P.-A. Hsieh, A. Rai, and M. Keil, "Addressing digital inequality for the socioeconomically disadvantaged through government initiatives: Forms of capital that affect ict utilization," Information Systems Research, vol. 22, no. 2, pp. 233-253, 2011.

[53] S. C. Srivastava and G. Shainesh, "Bridging the service divide through digitally enabled service innovations; evidence from indian health care service providers," MIS Quarterly, vol. 39, no. $1,2015$.

[54] M. Barrett, E. Davidson, J. Prabhu, and S. L. Vargo, "Service innovation in the digital age: Key contributions and future directions," MIS Quarterly, vol. 39, no. 1, pp. 135-154, 2015.

[55] N. Aimyong, Propensity score methods for competing risks. $\mathrm{PhD}$ thesis, The University of North Carolina at Chapel Hill, 2014.

[56] C. Zheng, R. Dai, P. Hari, and M.-J. Zhang, Instrumental Variable with Competing Risk Model, vol. 36. 032016. 